\title{
Childhood maltreatment and social functioning in adults with sub- clinical psychosis
}

\author{
David Boyda ${ }^{n}$, Danielle McFeeters \\ School of Psychology and Psychology Research Institute, University of Ulster at Magee, Northern Ireland
}

\begin{abstract}
a b s tract
Studies now acknowledge a robust association between childhood maltreatment and psychosis development in adulthood. Research shows that maltreatment not only influences the child's psychological wellbeing but also inhibits domains of social development. These social impairments have been found to predate the onset of psychosis and may crucially represent an intervening factor which triggers the decline towards psychosis. To examine social functioning as a potential mediating pathway between early maltreatment and sub-clinical psychosis. The study utilised data from the Adult Psychiatric Morbidity Survey (N1/47403). Psychotic-like experiences were assessed using the Psychosis Screening Questionnaire (PSQ) along with measures designed to capture childhood maltreatment and social impairment. Results revealed that maltreatment was associated with both social functioning deficits as well as psychotic symptomology. Furthermore, social functioning was found to mediate the relationship between maltreatment and psychosis. The results align with literature linking maltreatment to both social functioning deficits and psychosis. Crucially, the study bridges these research areas by presenting functional decline as possible risk indicator and intervening factor between maltreatment and psychosis. Intervention strategies should therefore seek to capitalise on treatments which boost social aptitude as a means of averting further decline towards psychopathology
\end{abstract}

\section{Introduction}

In recent years, numerous methodologically robust studies have established a link between distant trauma and psychosis (Cutajar et al., 2010). It has been suggested that the aetiology of psychosis in adulthood can be traced back to the crucial developmental period of early adolescence when the expression of psychosis becomes widespread (Escher et al., 2002). Exposure to trauma during this period is known to be associated with significant disruption to a child's psychological, emotional, and social development. While many negative experiences including peer victimisation, parental loss and economic hardship have been associated with the risk of developing psychotic-like experiences or symptoms; childhood maltreatment continues to impose the most distressing and prolonged effects (Bebbington, 2004; Fryers and Brugha, 2013; Janssen et al., 2004; Thompson et al., 2009; Varese et al., 2012). Although there has been enduring controversy surrounding the relationship between adverse childhood events and psychosis, recent studies have advocated support for a possible causal link. This is in light of the statistical association

\footnotetext{
${ }^{\mathrm{n}}$ Corresponding author.

E-mail address: David.Boyda@Gmail.com (D. Boyda)
}

between child sexual abuse (CSA) with subsequent psychotic disorders and schizophrenic syndromes in adulthood (Cutajar et al., 2010). Nonetheless, while research has largely focused on the consequences of childhood trauma and the elevated risk for psychiatric disorders; relatively little attention has been directed towards the effect that these experiences may exert on domains of social functioning (Roberts et al., 2004; Postmus, 2012).

Indeed, while trauma victims struggle to accept both the physical and psychological impact of their experience (including the loss of trust towards an emotionally significant person), there is now considerable evidence that these experiences also have a substantial impact on his or her social functioning over their life span (Cole and Putnam, 1992; Koenig et al., 2002; Haskett and Willoughby, 2007). Alink et al. (2012) found that children who experienced childhood trauma demonstrated poorer social functioning compared to nonabused children which intensified as the number of traumas increased. Other authors have found that childhood trauma is also associated with a greater likelihood of dysfunctional self-perception, poor self-esteem, fewer friends and social supports as well as disinterest in community involvement (Clemmons et al., 2007; Postmus, 2012). Moreover, trauma victims are more likely to isolate themselves from close social networks (i.e., family and friends) resulting in lower social support and limited social skills (Jehu et al., 1988).

Furthermore, a litany of studies now report that adult survivors frequently develop problems in interpersonal functioning including mistrust of others which can manifest in emotionally unstable and chaotic relationships (Dietrich, 2007). This may be a consequence of the timing of trauma in the developmental trajectory given that such maltreatment typically occurs at a time when beliefs and expectations are initially formed regarding interpersonal relationships. In sum, the impact of being sexually or physically abused, emotionally or physically neglected in childhood is likely to propagate into adulthood, manifesting in difficulties across domains of daily functioning. These factors are associated with poorer cognitive skills and reduced learning potential including the ability to work and financially sustain oneself (Postmus, 2012).

Indeed impairments in social functioning are a common feature in individuals at ultra-high risk (UHR) for psychotic disorder (Addington et al., 2008). Such impairments can impede functionality in domains necessary for normal daily activities such as selfcare, household activities, use of public transport and areas of interpersonal functioning such as communication and interaction with others (Viertiö et al., 2012). Furthermore, individuals with a psychotic disorder tend to be less independent, are less likely to be employed or in a partnership and are less satisfied with social relationships potentially increasing the risk of becoming socially isolated (Steiger, 1990). Prospective studies examining the behaviour of children genetically deemed high risk due to parental history of psychosis show patterns of social impairments which are thought to predict vulnerability to schizophrenia (Hans et al., 1992, 2000; Collip et al., 2013). In fact, recent studies have noted that impairments in social functioning often precedes the onset of psychosis (Ballon et al., 2007; Chudleigh et al., 2011; Ienciu et al., 2013; Kelleher et al., 2013) and as such may represent a useful subsyndromal marker. However, despite extensive literature linking childhood trauma and social impairment to psychosis; few studies if any have examined whether psychotic outcomes are contingent on the additional effect of poor social functioning resulting from early trauma.

In lieu of this, the current study sought to examine social functioning and loneliness as potential mediating pathways between early adverse experience 
and psychosis. First it was hypothesised that the experience of childhood trauma would significantly predict impairments across domains of social functioning and loneliness. Second, it was hypothesised that experiences of childhood trauma would be significantly and directly associated with psychotic like experiences. The final aim was to test whether the relationship between childhood trauma and psychotic like experiences would be mediated through dimensions of social functioning and loneliness after controlling for social and behavioural correlates known to pose a risk for psychosis.

\section{Methods}

\subsection{Sample}

The current study was based on the Adult Psychiatric Morbidity Survey (APMS) 2007. The APMS was designed to be representative of the population living in private households in England. Using the small users' postcode address file (PAF), the National Centre for Social Research adopted a multi-stage stratified probability sampling design. The survey consisted of a phase one and a phase two (clinical) interview. For phase one, 13,214 potentially eligible private households were identified. One adult aged 16 years or over was selected for interview within each household. Where there was more than one person aged 16 years or over, one adult was chosen randomly in order to ensure that all eligible members of any household had an equal chance of being selected. Fifty-seven per cent of eligible respondents agreed to participate which resulted in the completion of 7403 successful interviews (3197 males and 4206 females). Of those who did not take part, 31\% refused, 5\% were unable and the remaining $8 \%$ were not contactable. The phase one interview utilised standardised instruments to document demographic variables along with the assessment of common mental disorders.

\subsection{Weighting}

Data were weighted to take account of non-response so that the results were representative of the household population aged 16 years and over in England. Weighting occurred in three steps. First, sample weights were applied to take account of the different probabilities of selecting respondents in different sized households. Second, to reduce household non-response bias, a household level weight was calculated from a logistic regression model using interviewer observation and area-level variables (collected from Census 2001 data) available for responding and nonresponding households. Finally, weights were applied using the techniques of calibration weighting based on the age, sex and region to weight the data up to represent the structure of the national population, taking account of differential nonresponse between regions, and age-by-sex groups. The population control totals used were the Office for National Statistics (ONS) 2006 midyear household population estimates.

\subsection{Measures}

\subsubsection{Demographics}

Adjustment was made for a range of background variables known to be associated with psychotic-like experiences. These included age, gender and the following variables. Education: Educational attainment was initially assessed in a multi-level format ranging from no qualifications to degree level and above. This was recoded into a binary variable denoting whether or not respondents had any educational qualification: (0) No (1) Yes. Ethnicity: Ethnic background was coded into a binary variable: White (0) Ethnic minority (1). Low socio-economic status: Participants were asked if they were in receipt of state or housing benefits. The variable was coded into a binary variable: (0) "No" (1) "Yes". Drug dependence: Drug dependence was assessed by the agreement on any of the following drugs: "Dependent on cannabis, amphetamines, cocaine, crack, heroin/methadone, tranquilisers, or glue" and was summed as a composite variable then coded as a binary variable: (0) "Not dependent" (1) "Substance dependent".

\subsubsection{Mood disorder}

This study used two variables which identified the presence of anxiety and depression as specified by the Clinical Interview Schedule Revised (Lewiset al., 1992). The variables were summed and re-coded as a binary variable: (0) "The absence of a mood disorder" (1) "Presence of mood disorder".

\subsubsection{Childhood trauma}

A measure of physical and sexual abuse was derived from the domestic violence and abuse section of questionnaire.

2.3.3.1. Physical abuse. Experiences of physical abuse from parent or guardian before the age of 16 was generated from the item "Severely beaten by parent/step-parent/carer before the age of 16 ". Endorsed items were scored: (0) No, otherwise (1) Yes.

2.3.3.2. Sexual abuse. A measure of childhood sexual abuse was constructed from two items i.e. "Someone touched in sexual way without consent before the age of 16" and "Sexual intercourse without consent before the age of 16". Both items were combined and computed whereby a positive endorsement of one or both items were coded a (0) No otherwise (1) Yes.

2.3.3.3. Violence in the home. As part of the Stressful Life Events section individuals were provided with a list of events on a card and asked if you have ever experienced any of the events shown on the card. Two items were used to gauge exposure to violence in the home in childhood: "Violence in the home" and "When violence in the home last happened." Individuals who endorsed 0 for item one was classified as ( 0$)$ "No experience of violence". Those who endorsed a score of 1 were asked the follow up question "When violence in the home last happened?" Item two contained three response categories (1) "Within the last 6 months", (2) "More than 6 months but after 16 " (3) "More than months and before 16." Individuals who endorsed category 3 for item two were categorised as (1) "Yes, experience of violence before 16 ". All other responses to item two were denoted as missing.

2.3.3.4. Emotional neglect. A measure of emotional neglect was formed from responses to seven items contained with the social support section. Each contained three response categories (1) "Not true", (2) "Partly true", and (3) "Certainly true". The possible range of scores was between 7 and 21. Scores above the 90th percentile was considered indicative of individuals experiencing reasonably high levels of emotional neglect which was then coded as a binary variable: (1) "High emotional neglect" otherwise (0) "Low emotional neglect".

2.3.3.5. Parental separation. A new item relating to parental separation in childhood was computed from responses to the items "Lived with both natural parents until the age of 16 " and "Reason did not live continuously with both natural parents at home until the age of 16" (e.g. divorce, adoption, death of a parent). Respondents who endorsed yes for the initial item were coded as (0) "No parental separation". Those who answered no for the initial item were given the follow up question regarding the reason for not living continuously with both natural parents. Respondents who endorsed separation or divorce for item two were coded as (1) "Yes parental separation". All other reasons were computed as missing.

2.3.3.6. Neglect. Two items "Spent any time in any kind of institution up to the age of 16 " and "Ever taken into Local Authority Care as a child up to the age of 16 " were used to determine whether respondents experienced neglect in childhood. Responses to both items were scored as (0) No (1) Yes. If respondents endorsed one or both items they were classified as having experienced neglect in childhood. 


\subsubsection{Social functioning}

2.3.4.1. Social engagement. Social engagement was devised from the response to the items "Have difficulties in getting and keeping close relationships" and "Get on well with my friends and other relatives". The initial four point Likert scale was recoded so that higher scores reflected greater deficits i.e. (4) "Severe difficulties" (3) "Some difficulties" (2) "Occasional difficulties" (1) "No difficulties at all". These items were subsequently recoded into a binary variable. Scores of 4 endorsed on one or both of the relevant questions were classified as (1) "Yes". All others were designated (0) "No".

2.3.4.2. Recreational activities. A measure of recreational participation capturing both affective and behavioural elements was generated from the items "Enjoy my spare time" and involvement in clubs or associations. Respondents were asked if they were actively involved in either of the following: sports or sport supporters club; hobby or interest group. Responses for these items were coded as binary (0) "Not mentioned" or (1) "Mentioned". Respondents who endorsed being actively involved in either of these groups or scored either (1) "Very much" or (2) "Sometimes on the Enjoy my spare time" item were coded (1) "Yes, enjoy recreational participation", otherwise (0) "Do not enjoy recreational participation".

2.3.4.3. Activities in daily living (ADL). A composite measure of ADL was created from a combination of difficulties in: "Personal care", "Getting out and about or using transport", "Household activities", and "Managing money". The original response items (1) "No, no difficulty at all", (2) "Yes, some difficulty" and (3) "Yes, a lot of difficulty" were recoded as a binary variable. To score (1) "Yes, severe difficulties", individuals must have endorsed at least a 3 on any two items or a minimum of 2 on any three items. Combinations which reflected lower or no existing difficulties were demarcated (0) "No".

2.3.4.4. Loneliness. A single item "I feel lonely and isolated from other people" was used to represent a measure of loneliness. Items were scored on a 4-point Likert scale. Individuals who scored 3 (Some difficulties) or 4 (Severe difficulties) were coded: $1=$ Lonely' otherwise $0={ }^{\prime}$ Not lonely'.

\subsubsection{Psychotic-like experiences}

Psychotic-like experiences are considered 'attenuated' symptoms (Kelleher et al., 2013) can often present a risk for the subsequent decline towards clinically relevant psychosis. The criterion and construct validity of the nonclinical psychosis phenotype with schizophrenia demonstrate that it is a valid population which is to study the aetiology of psychosis (Kelleher et al., 2011). For the current study, the Psychosis Screening Questionnaire (Bebbington and Nayani, 1995) was used to assess psychotic like experiences in the past year. The PSQ comprises of five primary questions enquiring about mania, thought insertion, paranoia, strange experiences and hallucinations. Subsidiary items helped determine whether the responses were likely to signify true psychotic like experiences. The validity of these items has been outlined in the development of the PSQ. The current study employed these subsidiary items with the exception of the hallucination item which focused solely on more commonly encountered auditory experiences: (1b) 'People around you think it was strange' (2a) 'Came about in a way that many people would find hard to believe' (3b) 'Felt group of people was plotting to cause you serious harm' (4a) 'Was so strange that other people would find it very hard to believe' (5b) 'Heard voices saying quite a few words or sentences'. All items were coded as binary variables: (0) No (1) Yes, then computed to a final composite variable; (0) no PLE's (1) At least one PLE. Probable psychosis was intentionally excluded since the aim was to examine risk factors that may influence the expression of psychotic-like experiences.

\subsection{Analytic plan}

Analysis was conducted in three stages and involved a logistic mediation model with dichotomous outcomes (Muthén, 2011). First, the direct effects (c paths) from the trauma variables to psychotic-like experiences were estimated. Second, the mediating variables were introduced into the model and the direct effects between the trauma variables and mediators (a paths) and the mediators to the dependent variable (b paths) was estimated while controlling for the social functioning variables ( $c^{\prime}$ paths). Indirect effects were calculated using the product of two unstandardized paths linking the predictors (trauma) to the dependent variables (PSQ) through the social functioning mediators (e.g. $a_{1} \mathrm{X}$ $\mathrm{b}_{1}$ ). The model was specified and estimated in Mplus 7 (Muthén and Muthén, 2010) using robust maximum likelihood (MLR) estimator. To control for inflation of Type I errors associated with multiple testing, we adjusted the alpha-level, using the False Discovery Rate (FDC) procedure developed by Benjamini and Hochberg (1995). The proposed analytic model is depicted in Fig. 1.

\section{Results}

Descriptive statistics and weighted frequencies are shown in Tables 1 and 2. Males and females were almost equally represented (females $n=3801,51.4 \%$ ) with a mean age of 46 years. Twenty-four per cent reported that they had no academic qualifications and $12 \%$ of the sample was in receipt of state benefits. Those who classified themselves as an ethnic minority represented almost $14 \%$ of the sample and $27 \%$ reported that they had a mood disorder while $3.4 \%$ were drug dependent. The percentage of respondents who encountered any form of early trauma and met criteria for at least one psychotic symptom was consistently high (12.3-22.1\%).

The observed results showed a significant association between physical abuse ( $\mathrm{OR}=2.2)$ and social engagement. The results also showed significant associations between sexual abuse $(\mathrm{OR}=1.60)$, and $\mathrm{ADL}$ and loneliness $(\mathrm{OR}=1.70)$. Emotional neglect was significantly associated with three mediating variables; social engagement $(\mathrm{OR}=1.40)$, recreation $(\mathrm{OR}=0.45)$ and loneliness $(\mathrm{OR}=2.60)$. There were no significant associations between violence in the home and neglect and any of the mediating variables. There were significant associations between both ADL (OR=1.82) and loneliness (OR=2.0) with PSQ scores. There were no significant relationships between social engagement, recreation, and PSQ scores. The results are presented in Table 3.

The product of the estimates for the paths sexual abuse $\left(\mathrm{a}_{24}\right)$ on ADL and $\beta$ ADL on PSQ $\left(b_{3}\right)$ were positive and statistically significant $\left(\mathrm{a}_{24} \mathrm{~b}_{3} ; \quad=0.282\right.$, $95 \% \mathrm{CI}=0.005-0.514, \mathrm{p}=0.046)$. The product of the estimates for the paths sexual abuse $\left(\mathrm{a}_{30}\right)$, emotional neglect $\left(\mathrm{a}_{31}\right)$ on loneliness and loneliness on PSQ $\left(b_{4}\right)$ were also positive and statistically significant $\left(a_{30} b_{4} ;=0.312\right.$, 95\% CI $=0.102-0.522, \quad \mathrm{p}=0.000), \quad\left(\mathrm{a}_{31} \mathrm{~b}_{4} ; \quad \stackrel{\beta}{=}=0.636,95 \% \mathrm{CI}=0.339-0.932\right.$, $\mathrm{p}=0.000$ ) respectively. The results are presented in Table 4 .

\section{Discussion}

The current study sought to integrate two areas of research into one conceptual model by examining how different forms of childhood maltreatment may influence sub-clinical psychotic experiences through domains of social functioning.

The results from hypothesis one support other research which has found that children with a history of being sexually or physically abused or neglected often exhibit lower social competencies, are less socially accepted 
and lonelier than non-abused children (Rogosch et al., 1995; BlanchardDallaire and Hébert, 2014). Developing and maintaining positive and reciprocal social relationships involves among other things, an individuals' capacity to find peers who are trust worthy and will not exploit or abuse them (Kendall-Tackett, 2002). However, the ability to create and maintain relationships is affected by past abuse experiences. In fact, victims of child abuse often report reduced interpersonal trust compared to non-victims which may ultimately compromise the acquisition of stable and enduring social relationships (Blanchard-Dallaire and Hébert, 2014). Feelings of mistrust and suspicion of others may in part be due to negative schemas that abuse victims hold about others which are formed from their early adverse experiences; these are therefore likely to impinge

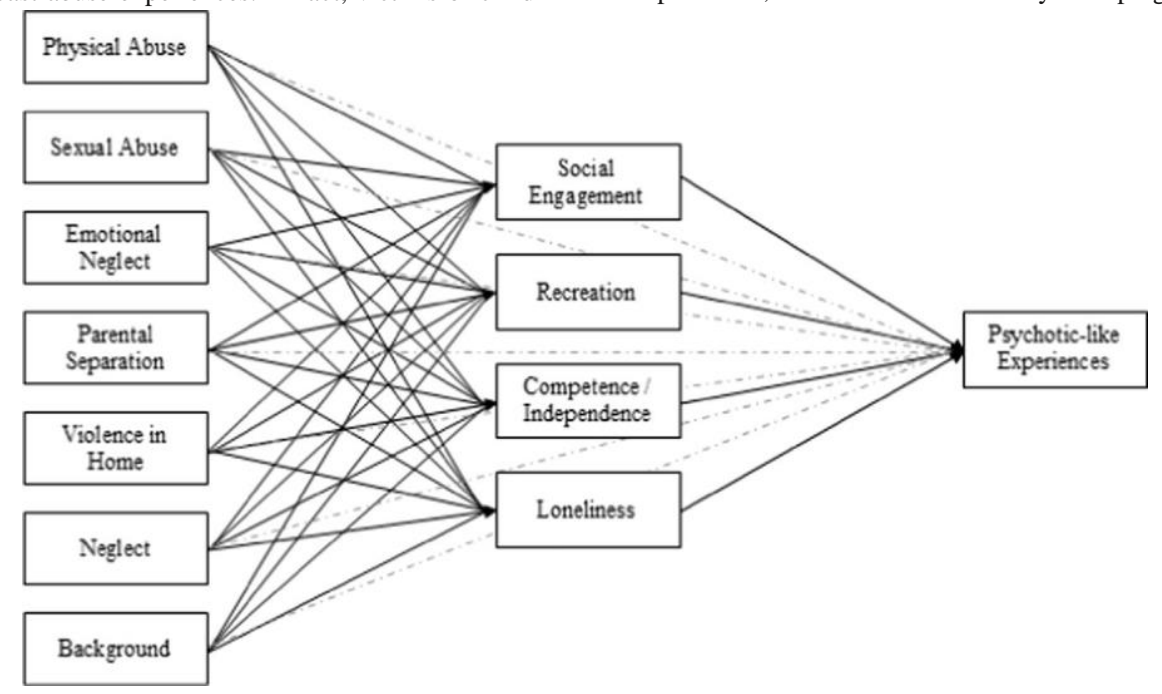

Fig. 1. Path model depicting the associations between trauma variables, social functioning, and PLEs.

Table 1

Descriptive statistics for the demographic correlates.

\begin{tabular}{|c|c|c|c|c|c|c|c|}
\hline & Age & Gender & Education & Ethnicity & Low SES & Mood disorder & Drug dep \\
\hline & & Female & No quals. & Minority & Yes & Yes & Yes \\
\hline M (S.D.) & $46(18.6)$ & \multicolumn{2}{|l|}{3801} & & & & \\
\hline$\%$ & & 51.4 & 24 & 13.7 & 12 & 27 & 3.4 \\
\hline \multicolumn{8}{|c|}{ *No quals.=No qualifications, Drug Dep⿳亠丷厂 Drug dependence. } \\
\hline \multicolumn{8}{|c|}{ Table 2} \\
\hline \multicolumn{8}{|c|}{ Weighted frequencies of childhood trauma and PSQ. } \\
\hline Items & Physical abuse & Sexual abuse & \multicolumn{2}{|c|}{ Parental separation } & Violence in home & Neglect & Emotional neglect \\
\hline Total & $345(4.7 \%)$ & $617(8.4 \%)$ & \multicolumn{2}{|c|}{$825(12.1 \%)$} & $230(3.3 \%)$ & $238(3.2 \%)$ & $900(12.2 \%)$ \\
\hline PSQ & $75(22.1 \%)$ & $91(14.9 \%)$ & \multicolumn{2}{|c|}{$108(13.1 \%)$} & $46(20.4 \%)$ & $41(17.5 \%)$ & $111(12.3 \%)$ \\
\hline
\end{tabular}

*Part one weights applied.

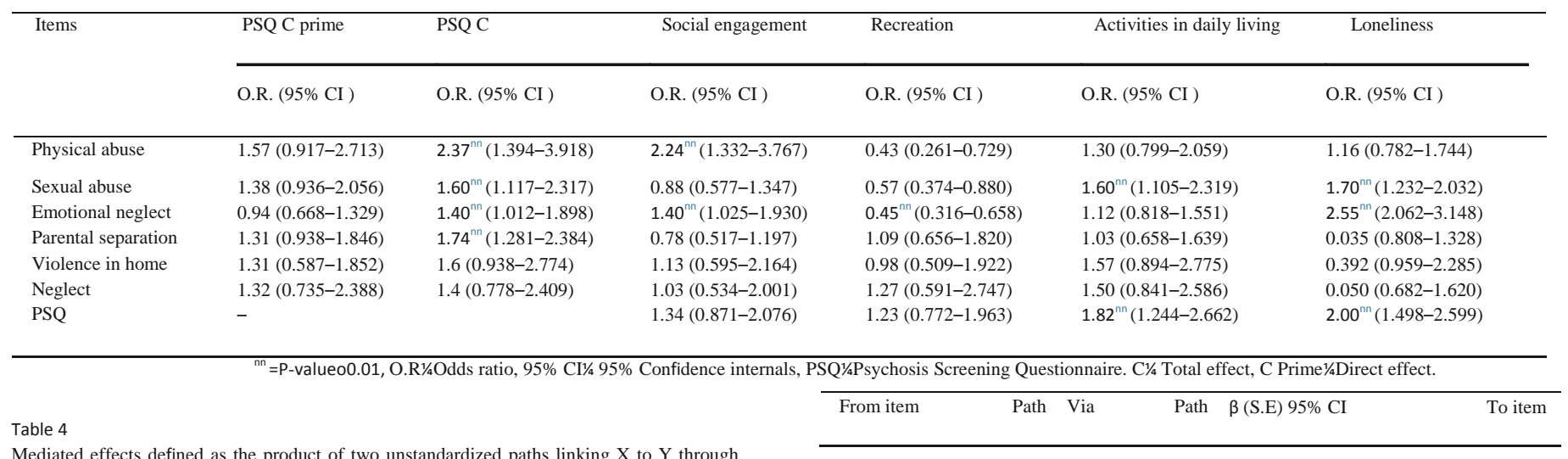

the mediators (e.g. $a_{1} b_{1}$ ). 


\begin{tabular}{|c|c|c|c|c|}
\hline & & $\overline{\mathrm{ADL}}$ & & \\
\hline Sexual Abuse & a24 & - & $b_{3}$ & $0.282(0.14) 0.005-0.514^{\mathrm{n}}$ PSQ \\
\hline Sexual abuse & a30 & - & $\mathrm{b}_{4}$ & $0.312(0.10) 0.102-0.522^{\mathrm{nn}}$ PSQ \\
\hline Emotional neglect & a31 & - & $\mathrm{b}_{4}$ & $\begin{array}{l}0.636(0.15) 0.339-\text { PSQ } \\
0.932^{\text {nn }}\end{array}$ \\
\hline
\end{tabular}

Confidence internals, PSQ $1 / 4$ Psychosis Screening Questionnaire. ${ }^{n}$ P-value $=0.05$.

${ }^{\mathrm{nn}}=\mathrm{P}$-valueo 0.01

upon their ability to connect in a recreational capacity with others (Jehu, 1992). Evidence also suggests that emotionally abused children are less competent in peer interactions and show fewer positive emotions which can increase the risk of peer rejection (Kinard, 1999). This is likely to generate feelings of loneliness, low self-esteem and aggression which may ultimately underlie deficits in social functioning including antisocial behaviours or a lack of pro-social behaviours (Ingersoll and Welfel, 2003). In relation to hypothesis two, the results support an emerging consensus linking childhood maltreatment with an increased risk of psychotic disorders or psychotic like experiences. All maltreatment experiences with the exception of violence in the home and neglect were found to predict PLE's which supports previous research (Bebbington, 2004; Read et al., 2005; Bebbington et al., 2011; Shevlin et al., 2011).

Finally, the third hypothesis that domains of social functioning and loneliness would mediate the associations between experiences of child abuse and psychosis was confirmed. The observed association corresponds with recent research linking alternative forms of trauma (IPV) to psychosis via loneliness (Boyda et al., 2014) and furthermore corroborates the view that affective and behavioural aspects of social functioning may represent important precursors to psychosis. Research evidence shows that child maltreatment typically occurs in the context of dysfunctional family relationships (Cash and Wilke, 2003) and it is likely that some parents who abuse or neglect their children also contribute to a generally negative family environment by virtue of an authoritarian, rigid, neglectful and chaotic approach to parenting (Mammen et al., 2002). Parental behaviours and interactions provide a template upon which children model their social behaviours and acquire the relevant knowledge to conduct their daily activities. However, disturbances to this relationship resulting from maltreatment may force the child to withdraw as a means of selfpreservation. As a result, this is likely to limit the child's opportunities to observe, mimic, and adopt their parent's behaviours which may ultimately relate to developmental delays across a range of skills required for normal day to day functioning. The detrimental effects of emotional neglect are wide and varied and have been associated with social isolation, difficulties in school, and feelings of despair (Collins and Gunnar, 1990; Crittenden, 1992). Loos and Alexander (1997) found that young adults, emotionally neglected as children, were more likely to experience loneliness and diminished peer interactions compared to their non-neglected peers thus coinciding with the results of this study.

In fact, research shows that abusive parents tend to perceive child rearing as less enjoyable and more difficult which may help explain why these individuals typically encourage the autonomy of their children less (Cicchetti and Lynch, 1993). In this way, the experience of early maltreatment may help precipitate the onset of decline in role functioning (e.g. cognitive and diminished selfsufficiency) (Clark et al., 2007) which in turn may have the additive effect of increasing the risk of developing psychotic symptomology.

While social functioning impairments are a central facet of psychosis (Bellack et al., 1990), identifying when and how these arise during course of the illness remains difficult (Stanghellini and Ballerini, 2002). Nevertheless, in line with a growing body of literature, the current findings indicate that impairments in social functioning may manifest long before the onset of psychotic symptoms and may accelerate decompensation to psychotic disorder in individuals with an increased risk for psychotic disorder (Chudleigh et al., 2011; Collip et al., 2013). As such, social functioning impairments may not simply be an epiphenomenon of psychosis but rather a "mediating indicator for vulnerability" (Nuechterlein et al., 1992).

\subsection{Limitations}

This study has several methodological limitations. First, this study is retrospective and cross-sectional thus inferences regarding causation between experiences of trauma, social functioning, and psychopathology should be acknowledged as speculative.

Second, the measurement of childhood trauma was conducted with a minimal number of items. Individuals who reported experiences of childhood maltreatment and subsequent psychopathology may have created biases (e.g., denial of severity of experiences, poor insight, social desirability biases etc.) which may potentially impede the assessment of the constructs under investigation. These considerations aside, the current study includes several strengths which merit recognition. The study involved a large nationally representative community sample with high levels of trauma as well as reasonably balanced gender split. Furthermore, rather than limiting the assessment of childhood trauma to one or two subtypes (i.e. physical and sexual abuse, neglect) this study explored a broader range of childhood adversities whilst controlling for a range of covariates known to influence social functioning and psychosis.

\subsection{Clinical implications}

The findings underscore the need for complete post-abuse evaluation that covers not only the most severe forms of maltreatment and trauma related symptoms but also incorporates an assessment of the child's social capabilities. That said it may prove difficult to focus treatment on any singular event given that the aetiology of psychotic experiences is complex whereby abusive experiences frequently co-occur and symptom expression is typically multivariate. However, timely treatment is especially important since early childhood to adolescence covers the developmental period during which social and role skills crystallise (Cornblatt et al., 2012). Relationship-based interventions may prove effective in overcoming poor interpersonal schemas and associated negative emotional states linked to relational memories (Pearlman and Courtois, 2005) while social skills training is reported to be effective in improving the social interactions of children who present with a variety of problems (Blanchard-Dallaire and Hébert, 2014). Given that social dysfunction may help identify an initial risk for psychosis, early detection tools coupled with appropriate intervention strategies which enhance social capacities may act as a buffer against poor mental health and abate the decline towards psychopathology in the long term.

\section{Conclusion}

Empirical reviews have established that severe childhood maltreatment is associated with a host of immediate and long-term sequelae which persist into adulthood (Davis et al., 2001). Since, childhood maltreatment is unlikely to transpire in isolation but instead co-occur in the context of other forms (Briere and Spinazzola, 2005) the findings emphasise the varied and additional adversities faced by maltreated children and demonstrate that young victims are at a higher risk of presenting impairments across varied domains of social functioning. The findings of the present study are in line with previous work that illustrates that childhood maltreatment has formerly been linked to decreased intellectual and cognitive functioning, deficits in pro-social behaviour, social withdrawal and isolation, as well as deficits in social competence with peers including avoidance of adults (Ingersoll and Welfel, 2003). Moreover, the findings also support other research which suggests that impaired social functioning may be an important risk factor for the development of psychosis (Cornblatt et al., 2012; Collip et al., 2013). To our 
knowledge this is the first study which has proposed a model whereby multiple risk factors for psychosis were examined together and when considered in its entirety, domains of social functioning may be deemed important intermediaries between previous experiences and normal psychological functioning.

\section{References}

Addington, J., Penn, D., Woods, S.W., Addington, D., Perkins, D.O., 2008. Social functioning in individuals at clinical high risk for psychosis. Schizophrenia Research 99, 119-124.

Alink, L.R.A., Cicchetti, D., Kim, J., Rogosch, F.A., 2012. Longitudinal associations among child maltreatment, social functioning, and cortisol regulation. Developmental Psychology 48, 224-236.

Ballon, J., Kaur, T., Marks, I., Cadenhead, K., 2007. Social functioning in young people at risk for schizophrenia. Psychiatry Research 151, 29-35.

Bebbington, P.E., 2004. Psychosis, victimisation and childhood disadvantage: evidence from the Second British National Survey of Psychiatric Morbidity. The British Journal of Psychiatry 185, 220-226.

Bebbington, P.E., Nayani, T., 1995. The psychosis screening questionnaire. International Journal of Methods in Psychiatric Research, 11-19.

Bebbington, P., Jonas, S., Kuipers, E., King, M., Cooper, C., Brugha, T., Meltzer, H., McManus, S., Jenkins, R., 2011. Childhood sexual abuse and psychosis: data from a cross-sectional national psychiatric survey in England. The British Journal of Psychiatry 199, 29-37.

Bellack, A.S., Morrison, R.L., Wixted, J.T., Mueser, K.T., 1990. An analysis of social competence in schizophrenia. The British Journal of Psychiatry 156, 809-818.

Benjamini, Y., Hochberg, Y., 1995. Controlling the false discovery rate: a practical and powerful approach to multiple testing. Journal of the Royal Statistical Society Series B (Methodological), 289-300.

Blanchard-Dallaire, C., Hébert, M., 2014. Social relationships in sexually abused children: self-reports and teachers' evaluation. Journal of Child Sexual Abuse 23, 326-344.

Boyda, D., McFeeters, D., Shevlin, M., 2014. Intimate partner violence, sexual abuse, and the mediating role of loneliness on psychosis. Psychosis.

Briere, J., Spinazzola, J., 2005. Phenomenology and psychological assessment of complex posttraumatic states. Journal of Traumatic Stress 18, 401-412.

Cash, S.J., Wilke, D.J., 2003. An ecological model of maternal substance abuse and child neglect: issues, analyses, and recommendations. American Journal of Orthopsychiatry 73, 392-404.

Chudleigh, C., Naismith, S.L., Blaszczynski, A., Hermens, D.F., Hodge, M.A.R., Hickie, I.B., 2011. How does social functioning in the early stages of psychosis relate to depression and social anxiety? Early Intervention in Psychiatry 5, 224-232.

Cicchetti, D., Lynch, M., 1993. Toward an ecological/transactional model of community violence and child maltreatment: consequences for children's development. Psychiatry Interpersonal and Biological Processes 56, 96-118.

Clark, R.E., Clark, J.F., Adamec, C., 2007. The encyclopedia of child abuse, 3rd edition Facts on File, New York.

Clemmons, J.C., Walsh, K., DiLillo, D., Messman-Moore, T.L., 2007. Unique and combined contributions of multiple child abuse types and abuse severity to adult trauma symptomatology. Child Maltreatment 12, 172-181.

Cole, P.M., Putnam, F.W., 1992. Effect of incest on self and social functioning: a developmental psychopathology perspective. Journal of Consulting and Clinical Psychology 60, 174-184.

Collins, W.A., Gunnar, M.R., 1990. Social and personality development. Annual Review of Psychology 41, 387-416.

Collip, D., Wigman, J.T., Lin, A., Nelson, B., Oorschot, M., Vollebergh, W.A.M., Ryan, J., Baksheev, G., Wichers, M., Os, J., van, Myin-Germeys, I., Yung, A.R., 2013. Dynamic association between interpersonal functioning and positive symptom dimensions of psychosis over time: a longitudinal study of healthy adolescents. Schizophrenia Bulletin 39, 179-185.

Cornblatt, B.A., Carrión, R.E., Addington, J., Seidman, L., Walker, E.F., Cannon, T.D., Cadenhead, K.S., McGlashan, T.H., Perkins, D.O., Tsuang, M.T., Woods, S.W., Heinssen, R., Lencz, T., 2012. Risk factors for psychosis: impaired social and role functioning. Schizophrenia Bulletin 38, 1247-1257.

Crittenden, P.M., 1992. Quality of attachment in the preschool years. Development and Psychopathology 4, 209-241.

Davis, J.L., Petretic-Jackson, P.A., Ting, L., 2001. Intimacy dysfunction and trauma symptomatology: long-term correlates of different types of child abuse. Journal of Traumatic Stress 14, 63-79.

Dietrich, A., 2007. Childhood maltreatment and revictimization: the role of affect dysregulation, interpersonal relatedness difficulties and posttraumatic stress disorder. Journal of Trauma \& Dissociation: the Official Journal of the International Society for the Study of Dissociation (ISSD) 8, 25-51.

Escher, S., Romme, M., Buiks, A., Delespaul, P., van Os, J., 2002. Formation of delusional ideation in adolescents hearing voices: a prospective study. American Journal of Medical Genetics 114, 913-920.

Fryers, T., Brugha, T., 2013. Childhood determinants of adult psychiatric disorder. Clinical Practice and Epidemiology in Mental Health 9, 1-50.

Hans, S.L., Auerbach, J.G., Asarnow, J.R., Styr, B., Marcus, J., 2000. Social adjustment of adolescents at risk for schizophrenia: the Jerusalem Infant Development Study. Journal of the American Academy of Child and Adolescent Psychiatry 39, 1406-1414.

Hans, S.L., Marcus, J., Henson, L., Auerbach, J.G., Mirsky, A.F., 1992. Interpersonal behavior of children at risk for schizophrenia. Psychiatry 55, 314-335.
Haskett, M.E., Willoughby, M., 2007. Paths to child social adjustment: parenting quality and children's processing of social information. Child: Care, Health and Development 33, 6777.

Ienciu, M.L., Bredicean, C.A., Romosan, F., Giurgi-Oncu, C.I., 2013. Social functioning in schizophrenia offspring. European Psychiatry 28, 1.

Ingersoll, R.E., Welfel, E.R. (Eds.), 2003. The Mental Health Desk Reference: A PracticeBased Guide to Diqgnosis, Treatment, and Professional Ethics: A Practice-Based Guide to Diagnosis, Treatment, and Professional Ethics, New edition. John Wiley \& Sons, New York, Chichester.

Janssen, I., Krabbendam, L., Bak, M., Hanssen, M., Vollebergh, W., Graaf, R., Os, J., 2004. Childhood abuse as a risk factor for psychotic experiences. Acta Psychiatrica Scandinavica 109, 38-45.

Jehu, 1992. Adult survivors of sexual abuse. In: Ammerman, Hersen (Ed.), Assessment of Family Violence: A Clinical and Legal Sourcebook. Wiley, New York, pp. 348-370.

Jehu, D., Gazan, M., Klassen, C., 1988. Beyond Sexual Abuse: Therapy with Women Who Were Childhood Victims, Wiley Series on Psychotherapy and Counselling. John Wiley \& Sons, Oxford, England.

Kelleher, I., Devlin, N., Wigman, J.T., Kehoe, A., Murtagh, A., Fitzpatrick, C., Cannon, M., 2013. Psychotic experiences in a mental health clinic sample: implications for suicidality, multimorbidity and functioning. Psychological Medicine, 1-10.

Kelleher, I., Harley, M., Murtagh, A., Cannon, M., 2011. Are screening instruments valid for psychotic-like experiences? a validation study of screening questions for psychotic-like experiences using in-depth clinical interview. Schizophrenia Bulletin 37, 362-369.

Kendall-Tackett, K., 2002. The health effects of childhood abuse: four pathways by which abuse can influence health. Child Abuse \& Neglect 26, 715-729.

Kinard, E.M., 1999. Perceived social skills and social competence in maltreated children. The American Journal of Orthopsychiatry 69, 465-481.

Koenig, A.L., Ialongo, N., Wagner, B.M., Poduska, J., Kellam, S., 2002. Negative caregiver strategies and psychopathology in urban, African-American young adults. Child Abuse \& Neglect 26, 1211-1233.

Lewis, G., Pelosi, A.J., Araya, R., Dunn, G., 1992. Measuring psychiatric disorder in the community: a standardized assessment for use by lay interviewers. Psychological Medicine 22, 465-486.

Loos, M.E., Alexander, P.C., 1997. Differential effects associated with self-reported histories of abuse and neglect in a college sample. Journal of Interpersonal Violence 12, 340-360.

Mammen, O.K., Kolko, D.J., Pilkonis, P.A., 2002. Negative affect and parental aggression in child physical abuse. Child Abuse \& Neglect 26, 407-424.

Cutajar, MC, Mullen, PE, Ogloff, JP, Thomas, SD, Wells, DL, Spataro, J, 2010. Schizophrenia and other psychotic disorders in a cohort of sexually abused children. Archives of General Psychiatry 67, 1114-1119.

Muthén, B.O., 2011. Applications of Causally Defined Direct and Indirect Effects in Mediation Analysis using SEM in MPlus.

Muthén, L.K., Muthén, B.O., 2010. Mplus User's Guide, Fifth Edit., ed Muthen \& Muthen, Los Angeles, CA.

Nuechterlein, K.H., Dawson, M.E., Gitlin, M., Ventura, J., Goldstein, M.J., Snyder, K.S., Yee, C.M., Mintz, J., 1992. Developmental processes in schizophrenic disorders: longitudinal studies of vulnerability and stress. Schizophrenia Bulletin 18, 387-425.

Pearlman, L.A., Courtois, C.A., 2005. Clinical applications of the attachment framework: Relational treatment of complex trauma. Journal of Traumatic Stress 18, 449-459.

Postmus, J., 2012. Sexual violence and abuse: an encyclopedia of prevention, impacts, and recovery. ABC-CLIO.

Read, J., Van Os, J., Morrison, A.P., Ross, C.A., 2005. Childhood trauma, psychosis and schizophrenia: a literature review with theoretical and clinical implications. Acta Psychiatrica Scandinavica 112, 330-350.

Roberts, R., OConnor, T., Dunn, J., Golding, J., 2004. The effects of child sexual abuse in later family life; mental health, parenting and adjustment of offspring. Child Abuse \& Neglect 28, 525-545.

Rogosch, F.A., Cicchetti, D., Aber, J.L., 1995. The role of child maltreatment in early deviations in cognitive and affective processing abilities and later peer relationship problems. Development and Psychopathology 7, 591-609.

Shevlin, M., Murphy, J., Read, J., Mallett, J., Adamson, G., Houston, J.E., 2011. Childhood adversity and hallucinations: a community-based study using the National Comorbidity Survey Replication. Social psychiatry and psychiatric epidemiology 46, 1203-1210.

Stanghellini, G., Ballerini, M., 2002. Dis-sociality: the phenomenological approach to social dysfunction in schizophrenia. World Psychiatry: Official Journal of the World Psychiatric Association (WPA) 1, 102-106.

Steiger, J.H., 1990. Structural model evaluation and modification: An interval estimation approach. Multivariate Behavioral Research 25, 173-180.

Thompson, J.L., Kelly, M., Kimhy, D., Harkavy-Friedman, J.M., Khan, S., Messinger, J.W., Schobel, S., Goetz, R., Malaspina, D., Corcoran, C., 2009. Childhood trauma and prodromal symptoms among individuals at clinical high risk for psychosis. Schizophrenia Research 108 , 176-181.

Varese, F., Smeets, F., Drukker, M., Lieverse, R., Lataster, T., Viechtbauer, W., Read, J., Os, J. van, Bentall, R.P., 2012. Childhood adversities increase the risk of psychosis: a meta-analysis of patient-control, prospective- and cross-sectional cohort studies. Schizophrenia Bulletin 38, 661-671.

Viertiö, S., Tuulio-Henriksson, A., Perälä, J., Saarni, S.I., Koskinen, S., Sihvonen, M., Lönnqvist, J., Suvisaari, J., 2012. Activities of daily living, social functioning and their determinants in persons with psychotic disorder. European Psychiatry 27, 409-415. 